\title{
SIBERIAN RADIOHELIOGRAPH: FIRST RESULTS
}

\author{
S.V. Lesovoi ${ }^{1}$, A.T. Altyntsev ${ }^{1}$, A.A. Kochanov ${ }^{1}{ }^{1}$, V.V. Grechnev ${ }^{1}$, A.V. Gubin ${ }^{1}$, D.A. Zhdanov ${ }^{1}$, \\ E.F. Ivanov ${ }^{1}$, A.M. Uralov ${ }^{1}$, L.K. Kashapova ${ }^{1,2}$, A.A. Kuznetsov ${ }^{1}$, N.S. Meshalkina ${ }^{1}$, R.A. Sych ${ }^{1}$ \\ ${ }^{I}$ Institute of Solar-Terrestrial Physics SB RAS, Irkutsk, Russia \\ ${ }^{2}$ Irkutsk State University, Irkutsk, Russia \\ svlesovoi@gmail.com,altyntsev@iszf.irk.ru,kochanov@iszf.irk.ru,grechnev@iszf.irk.ru,gubin@iszf.irk.ru, \\ zhdanov@iszf.irk.ru,eugenessrt@gmail.com,uralov@iszf.irk.ru,lkk@iszf.irk.ru, \\ a_kuzn@iszf.irk.ru,nata@iszf.irk.ru,sych@iszf.irk.ru
}

Regular observations of active processes in the solar atmosphere have been started using the first stage of the multiwave Siberian Radioheliograph (SRH), a T-shaped 48-antenna array with a 4-8 GHz operating frequency range and a $10 \mathrm{MHz}$ instantaneous receiving band. Antennas are mounted on the central antenna posts of the Siberian Solar Radio Telescope. The maximum baseline is $107.4 \mathrm{~m}$, and the angular resolution is up to 70 ". We present examples of observations of the solar disk at different frequencies, "negative” bursts, and solar flares. The sensitivity to compact sources reaches 0.01 solar flux units $\left(\approx 10^{-4}\right.$ of the total solar flux) with an accumulation time of about 0.3 s. The high sensitivity of SRH enables monitoring of solar activity and allows studying active processes from characteristics of their microwave emission, including faint events, which could not be detected previously.

Keywords Sun $\cdot$ Radio emission $\cdot$ Solar flares $\cdot$ Radio telescopes $\cdot$ Negative bursts

\section{INTRODUCTION}

The Institute of Solar-Terrestrial Physics of Siberian Branch of the Russian Academy of Sciences has developed the largest complex of instruments for ground-based observations of various space weather phenomena and their sources - from events occurring in the solar atmosphere to ionospheric plasma disturbances. A prominent place in this complex, as well as in the international cooperation, belongs to the instrumentation for monitoring and diagnostics of solar active processes by radio astronomy methods. Information supplied by radio observations is significant for both fundamental solar physics and practical use, especially in Russia which still lacks continuous observations of solar activity from space.

The radio telescopes are located in the Radioastrophysical Observatory (RAO) (the Badary area, the Buryat Republic). Solar observations are made during daylight hours from 00 to 10 UT in summer and from 02 to 08 UT in winter. The observatory located away from communities, the radio noise level is low. In RAO, a multiwave Siberian Radioheliograph (SRH, Figure 1) is under construction. Total-flux spectropolarimeters observe solar emission in a frequency band from $50 \mathrm{MHz}$ to $24 \mathrm{GHz}$. Data from the Badary Broadband Microwave Spectropolarimeter (BBMS) in the 4-8 GHz operating frequency band of the first stage of SRH are also available [Zhdanov, Zandanov, 2015]. 
Observational data from RAO's radio telescopes are used by Russian and foreign scientists for studies of fundamental problems in solar physics, including those carried out under international programs. Prospects for the use of the observational data to solve applied problems determine the significant contribution of the Fedorov Institute of Applied Geophysics, Roshydromet, into the development of the radio telescope complex. Solar activity data, including real-time ones, are available online at http://badary.iszf. irk.ru

Radio observations provide unique information on processes of plasma heating and particle acceleration in the solar atmosphere. The total solar flux at a frequency of $2.8 \mathrm{GHz}$ (the so-called $10.7 \mathrm{~cm}$ index) is the most objective assessment of the current level of solar activity and its variability over several solar cycles. The $10.7 \mathrm{~cm}$ index is an input parameter in various models of near-Earth space, magnetosphere and ionosphere.

The advantage of solar activity monitoring in the radio range is its all-weather capability, as weather conditions have little effect on a received signal. The cost of radio telescopes is relatively low compared to space missions and optical instruments. Solar plasma transparency for radio emission increases with its frequency. The atmospheric transparency window enables ground-based observatories to receive solar radio emission from tens of $\mathrm{MHz}$ to hundreds of $\mathrm{GHz}$ and to gain information from emitting areas from the chromosphere to the high corona. The range of the time scales under study is also extensive, from millisecond pulses generated by coherent mechanisms in abnormally bright compact sources to multi-year solar cycles.

Some physical parameters of the solar corona can only be estimated from radio observations. The geoeffective potential of eruptions of solar magnetized plasma, which cause sporadic disturbances in near-Earth space, depends on coronal magnetic fields; the rate and intensity of eruptive processes are determined by dynamics of magnetic energy release and its conversion into energy of plasma particles and emission. Therefore, one of the most important tasks is to monitor the evolution of magnetic structures in the solar corona. The brightness of magnetically active lines in the corona is too low against the photospheric background that hampers magnetic field measurements in the corona with optical instruments. Microwave observations are capable of solving this problem, because the microwave spectrum and polarization strongly depend on the strength and orientation of the magnetic field in a source. Radio magnetography of the solar corona becomes possible with the advent of new generation radioheliographs which provide sequences of solar disk images at different frequencies (see, for example, [Lang et al., 1993; Wang et al., 2015]). An important feature of radio observations is their high sensitivity to emission of nonthermal electrons. Radio observations are sensitive enough to nonthermal emission from coronal regions where the plasma density is too low to produce detectable hard X-rays.

Observations of eruptive processes in the solar corona are important to improve physical background of forecasting and diagnostics of geoeffective phenomena such as solar flares and coronal mass ejections. Radioheliographs detect microwave bursts associated with energy release processes and reveal their sources on the solar disk.

Flare processes often produce radio bursts whose intensity is millions of times higher than background. Data on their spectrum and sources are important for diagnostics of sporadic solar activity, which is necessary to analyze causes of malfunctions in electronic systems on spacecraft, radars, navigation and communication systems. Generalization and systematization of these data have important implications for hardware developers. 


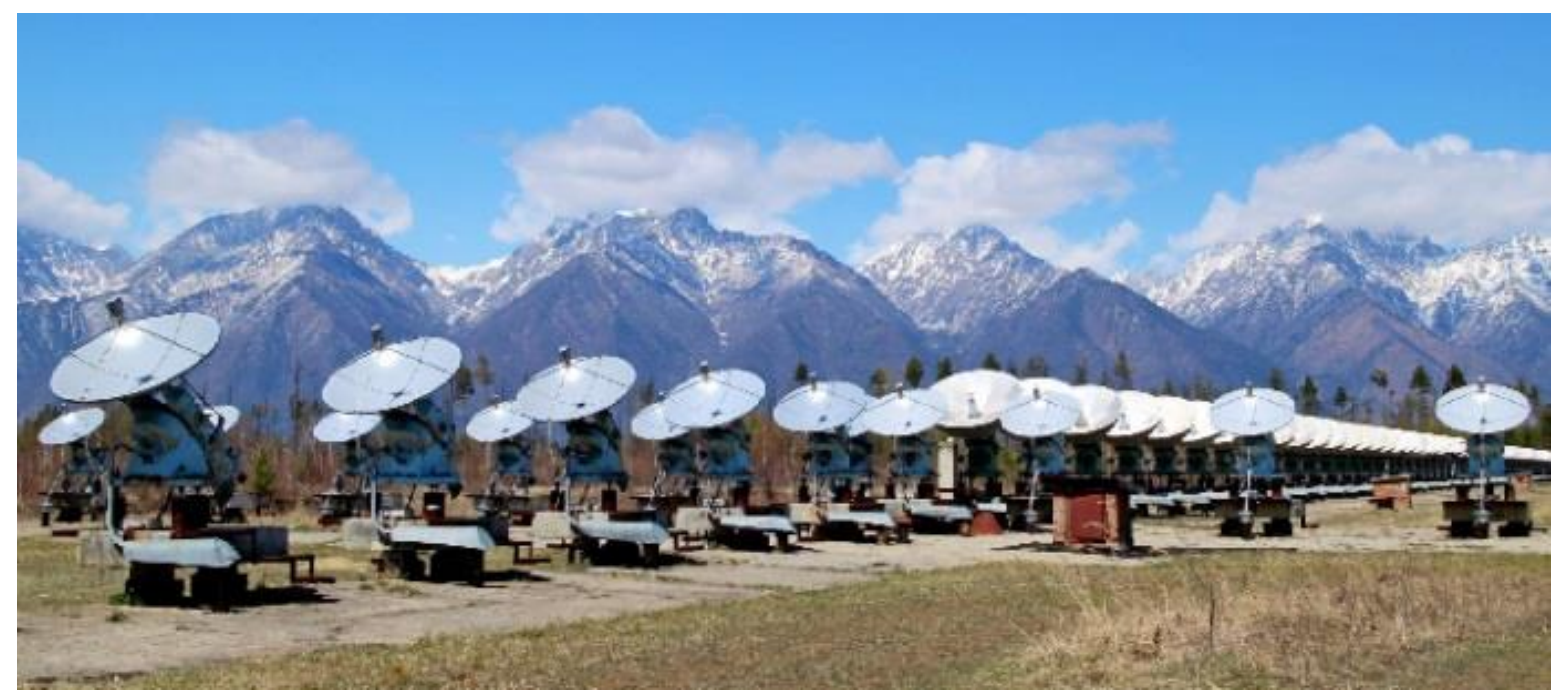

Figure 1. The central part of the SRH antenna array and the northern arm of SSRT

Objectives of observations in the microwave range involve identifying predictors of powerful solar flares, excitation of shock waves, and appearance of accelerated electron and proton fluxes in interplanetary space.

Since the angular resolution of a telescope depends on the ratio of received emission wavelength to its aperture, observation of the evolution of active regions and flare sources requires developing instruments of hundreds of meters in size. To reach the above objectives, it is important to combine sufficiently high spatial resolution with a wide field of view exceeding the solar disk that is possible to attain with multi-element interferometers. Their spatial resolution depends on the aperture of the antenna array; and the field of view is determined by individual antenna elements. Spectral resolution should be high enough to identify emission mechanisms, regions with optically thin and thick emission, characteristics of emitting electrons, etc.

Important information on emission mechanisms is carried by polarization, insufficiently used so far to interpret observations because of its dependence on the spatial structure of sources. The analysis of polarization data is often complicated by propagation effects of the emission from the source in surrounding plasma. These effects cause absorption, polarization reversal, and scattering. On the other hand, if these effects are significant, they allow estimating plasma parameters on the way from the source to an observer.

Until recently, the main instrument of the ISTP SB RAS Radioastronomical complex was SSRT, which commenced observations in 1986 [Smolkov et al., 1986; Grechnev et al., 2003]. SSRT is included in Russia's list of unique instruments, and the team of designers got the Russian Federation Government Award. A large amount of digital data have been accumulated from daily observations at a frequency of $5.7 \mathrm{GHz}$ during daylight hours in the form of two-dimensional radio maps and one-dimensional distributions of radio brightness. At present, SSRT is transformed into a new instrument, multiwave Siberian Radioheliograph [Lesovoi et al., 2014]. In 2016, the firststage 48-antenna array (SRH-48) commenced observations in a frequency band of 4-8 GHz.

Previously, concurrent solar imaging was possible at three frequencies: $5.7 \mathrm{GHz}$ at SSRT, 17 and $34 \mathrm{GHz}$ at the Japanese Nobeyama Radioheliograph (NoRH) [Nakajima et al., 1995]. The observational daytime interval of NoRH from 23 to 06 UT has a large overlap with that of SSRT. There is also an overlap with the RATAN-600 radio 
telescope, which measures one-dimensional radio brightness distribution over the solar disk in a wide frequency range with a high spectral resolution [Bogod, 2011; Kaltman et al., 2015]. Multiwave observations provide a wealth of information on the quiet solar atmosphere and active processes in it; the joint analysis of microwave observations with data from different spectral ranges gives a unique insight into various solar processes, their interrelation and effect on near-Earth space (see, e.g., [Altyntsev, Kashapova 2014]). The current state of digital electronics and computer technology makes it possible to develop multi-element radio interferometers producing solar images at a number of frequencies almost simultaneously.

Current implementation of several projects confirms that new-generation radioheliographs are required. In the microwave range, the largest radio telescopes (with a baseline of about one kilometer or more) are the American Frequency Agile Solar Radiotelescope (FASR) [Bastian et al., 1998] and the Chinese Mingantu Ultrawide Spectral Radioheliograph (MUSER) [Yan et al., 2009]. The projects are currently at different stages of implementation. Under the FASR project, the Expanded Owens Valley Solar Array (18 antennas, 1-9 GHz) is employed to devise and test radioheliograph systems. Efforts are underway to increase the number of antennas and to expand the receiving band up to 2.5-18 GHz [Gary et al., 2012]. In MUSER, located in Inner Mongolia, antenna systems of CSRH-I (40 antennas, 0.4-2.0 GHz) and CSRH-II (60 antennas, 2-15 GHz) have been installed. Test observations at some frequencies have started. Efforts are applied to phase calibration issues.

This paper presents preliminary results of observations made with the first stage of SRH with the T-shaped 48antenna array in the 4-8 GHz frequency range. Single-frequency test observations were made since early 2016. Since July 2016, SRH-48 has routinely been observing at five frequencies. During this period, solar activity was low. This allowed us to assess capabilities of the new instrument to study faint events, which cannot be detected by total-flux telescopes.

\section{CHARACTERISTICS OF THE SIBERIAN RADIOHELIOGRAPH WITH THE 48-ANTENNA ARRAY}

The Siberian Solar Radio Telescope, which is transformed into the Siberian Radioheliograph, has the following characteristics [Grechnev et al., 2003]. SSRT is a cross-shaped interferometer consisting of two EW and SN arrays, each with 128 antennas of $2.5 \mathrm{~m}$ in diameter. The baseline is as long as $622.3 \mathrm{~m}$, which determines the angular resolution up to $21^{\prime \prime}$ in two-dimensional images and up to $15^{\prime \prime}$ in one-dimensional radio brightness distributions. Both circular polarized components (RCP and LCP) are measured. The $14 \mathrm{~ms}$ period of polarization modulation determines the highest temporal resolution of one-dimensional measurements. In the two-dimensional mode, images are formed as a result of the change of the Sun's position relative to interference maxima, firstly, because of the simultaneous receiving at different frequencies in the 5.67-5.79 GHz band and, secondly, because of Earth's diurnal rotation. The latter determines the transit time of the interference maximum across the Sun; therefore SSRT can produce images every 2-3 minutes at most.

The Siberian Radioheliograph uses an essentially different imaging principle, Fourier synthesis [Lesovoi et al., 2014]. The temporal resolution determined by the receiver system is much higher. At present, routine observations are performed using the 48-antenna array with antennas installed on the central posts of SSRT along the east, west, and south arms. Adjustment of SRH-48 systems is still in progress. The observing frequencies, each of the $10 \mathrm{MHz}$ bandwidths in 
the 4-8 GHz range, are set by software. The time to switch from one frequency to another is currently about $2 \mathrm{~s}$, and the accumulation time at each frequency is $0.28 \mathrm{~s}$ for each circularly-polarized component. The maximum baseline is 107.4 $\mathrm{m}$, and the spatial resolution is as high as 70" at $8 \mathrm{GHz}$. Both circularly-polarized components are measured. The sensitivity to compact sources reaches $10^{-4}$ of total solar flux [Lesovoi, Kobets, 2017].

The number of observing frequencies can be changed depending on the observational program. For quasistationary objects such as sunspot-associated sources, several hundreds of frequencies can be used to achieve a desired spectral resolution. Observations of flares require high temporal resolution, for which the number of frequencies can be reduced. Since 1 July 2016, observations have been made at five frequencies - 4.5, 5.2, 6.0, 6.8, and 7.5 GHz. Raw data are available at [ftp://badary.iszf.irk.ru/data/srh48]. Software has been developed and tested to produce raw solar images, clean them, and calibrate in brightness temperature units. File formats for storage of data and software for their remote access are being developed.

Along with observations at SRH-48, the rest of SSRT's antennas continue observations at $5.7 \mathrm{GHz}$ in the original operating mode. All antennas of the north SSRT arm and outer antennas of other arms provide two-dimensional images of compact structures in the solar atmosphere with a resolution of up to 21 " and a 2-3 min interval. Daily maps in intensity and circular polarization near local noon are available at [ftp://badary.iszf.irk.ru].

To monitor solar activity and main SRH systems, the so-called correlation plots are used. They represent a proxy of radio flux and display temporal variation in the sum of cross-correlations of all antenna pairs. Methods for calculating the correlation plots and their relation with characteristics of solar emission are discussed in [Lesovoi, Kobets, 2017]. Changes in the correlation plots are associated with variations both in the brightness of sources and in their structure. Real-time correlation plots are accessible online at [http://badary.iszf.irk.ru/srhCorrPlot.php].

The correlation plot for 9 August 2016 in Figure 2 is interesting due to the presence of three negative bursts and subsequent C2.5 flare in active region 12574 (N04E59). Maintenance of SRH systems was carried out during first hours of that day. Observations started at 02:45 UT.

Figure 3 exemplifies a set of solar images observed at five frequencies in the right-handedly and left-handedly polarized emission without cleaning the images from the contribution of side lobes of the interferometer. A bright source on the north-west is a response to active region NOAA 12571. Side lobes look like narrow zebra stripes. The images at frequencies below $6 \mathrm{GHz}$ also show a brightening on the east limb, which is associated with rising active regions. The field of view of the images is one-third larger than the solar disk. The apparent size of a bright compact source decreases with frequency, being determined by convolution of the real source with the frequency-dependent interferometer beam.

To calibrate images in brightness temperatures, the technique employed at NoRH and SSRT has been elaborated. For each image, histograms of the brightness distributions are computed for the regions occupied by the sky and the solar disk. The difference between the positions of the histograms' peaks represents the brightness temperature of the quiet Sun [Kochanov et al., 2013]. This temperature, decreasing with increasing frequency, is referred to the measurements made in [Zirin et al., 1991; Borovik, 1994]. In particular, at the five SRH frequencies, the values of $18.7,17.1,15.4,14.3$, and 13.5 thousand Kelvin are adopted. Brightness temperatures of compact sources can be considerably lower than the real temperatures if the source is less than the beam pattern of SRH-48. 


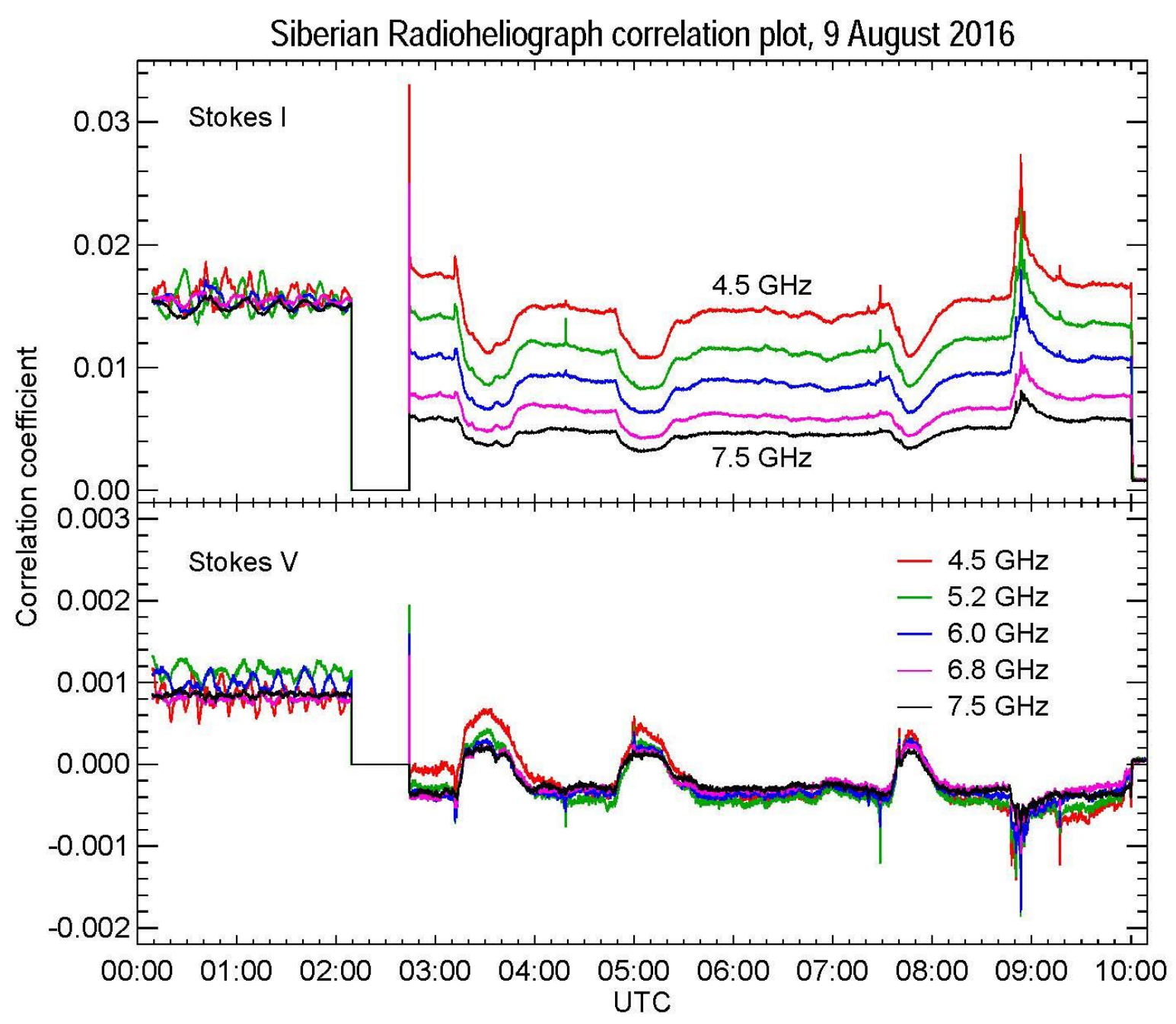

Figure 2. Correlation plots with three negative bursts and a C2.5 flare at 08:55 for 9 August 2016.

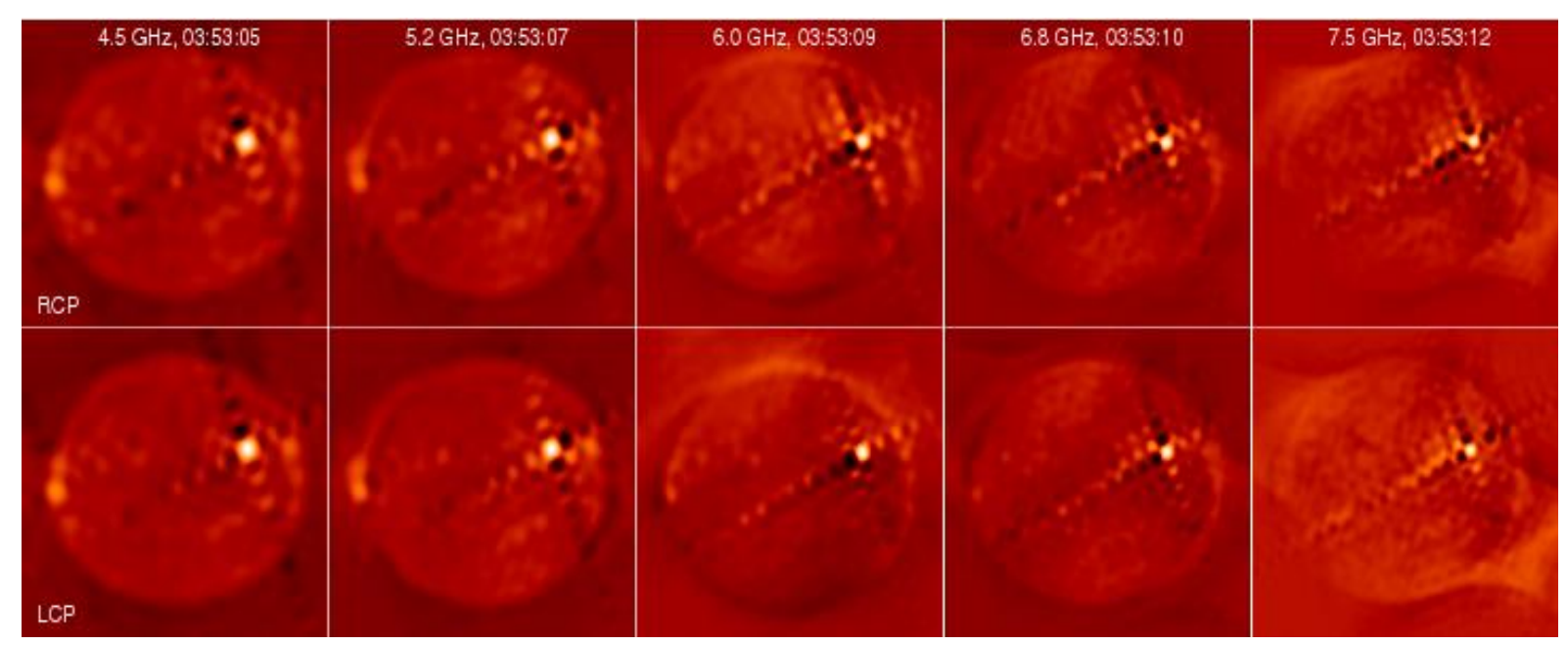

Figure 3. Images of the solar disk at five frequencies for the 9 June 2016 in the circularly-polarized right (RCP) and left (LCP) emissions 


\section{PRELIMINARY RESULTS OF FIRST OBSERVATIONS}

Temporary depressions of radio flux below the quasistationary level (negative bursts) sometimes occur. They are caused by screening of emission from compact radio sources or quiet solar regions in low-temperature plasma ejected into the solar corona during eruptions. The dependence of the absorption depth on the emission frequency and properties of absorbing plasma allows estimating the temperature, density, and size of the eruptive structure if a negative burst is recorded at several radio frequencies [Grechnev et al., 2013]. Negative bursts provide valuable information about eruptive events, but they are very rarely observed. The maximum number of negative bursts recorded by all ground-based stations during a year was as small as 14 in 1991 . The occurrence of three negative bursts during one day on August 9, 2016 is unprecedented (Figure 2).

SRH images in Figure 4 show that these negative bursts were caused by a decrease in the brightness of the northern source near the east limb. Its maximum brightness temperature near the minimum of the first negative burst in Figure 4, $a$ was 0.22 MK. After the end of the negative burst, the brightness temperature of the screened source increased to $0.53 \mathrm{MK}$ (Figure 4,b), whereas in the other three sources its change did not exceed $13 \%$. The last value is the upper boundary of the total error in measurements of brightness temperatures from SRH data caused by both the calibration instability and flat-field issues. The brightness temperatures in the images are lower than those typically observed because of insufficient spatial resolution of the current configuration of SRH-48.

Figure 5 presents total flux time profiles of the screened microwave source in total intensity and polarization computed from 2537 pairs of images observed with an interval of 8.4 s. The comparison with the total flux at 3.75 $\mathrm{GHz}$, recorded by NoRP with an interval of $1 \mathrm{~s}$, demonstrates the solar origin of the negative bursts and high sensitivity of SRH. With intensity depressions reaching $-5 \mathrm{sfu}\left(1 \mathrm{sfu}=10^{-22} \mathrm{~W} /\left(\mathrm{m}^{2} \mathrm{~Hz}\right)\right)$ at $5.2 \mathrm{GHz}$ the polarization shifted to positive values. This corresponds to screening of the left-polarized source (Figure 4, $c, d$ ).

No coronal mass ejections were associated with these negative bursts and no solar observations were made by SDO/AIA on that day. Rising jets (surges) were found in $304 \AA$ images produced by the STEREO-A space observatory located in the Earth orbit to the east of it by $152^{\circ}$. The images in Figure 6 are negatives of variance maps revealing all changes in the images. Each pixel of such a map represents variance of values at this point in all images taken within a certain time interval [Grechnev, 2003]. The frame denotes the region whose flux variations are shown in Figure $5 d$. The occurrence of negative bursts is certainly related to the surges screening the microwave source. Further analysis of these negative bursts promises estimating parameters of surges.

Along with high sensitivity, the SRH receiving system has a sufficiently wide dynamic range to observe the microwave emission from flares without attenuators. Figure 7 shows correlation plots with three powerful M-class flares: M5.0 (02:11), M7.6 (05:16), and M5.5 (05:31 UT) recorded on 23 July 2016. The most intense was the microwave burst in the last flare whose flux at SRH frequencies exceeded 800 sfu. Between 05:28:30 and 05:30:30 UT, polarization reversal occurred within the SRH frequency range (Figure 8). The polarization reversal of flare microwave emission can be caused by different reasons. To determine them, further analysis of spatially-resolved data is required. 
Figure 9 presents correlation plots of the 18 April 2016 M7.6 flare. With a general correspondence between the SRH (Figure 9, $a$ ) and NoRH (Figure 9, b,c) data, the burst time profile, as usual, becomes sharper with increasing frequency. This event is interesting due to pronounced broadband microwave pulsations with a period of about $30 \mathrm{~s}$.

As a rule, the 4-8 GHz receiving band of SRH does not cover the entire spectrum of gyrosynchrotron emission of powerful solar flares, which may extend to several tens of GHz. Nevertheless, SRH images allow identifying important flare structures, for example, high flare loops invisible at high frequencies [Altyntsev et al., 2016; Fleishman et al., 2016]. In most weak to moderate microwave bursts, the spectral peak falls within the SRH frequency band or lies close to it [Nita et al., 2004].

A joint analysis of SRH observations with data obtained in different spectral ranges is particularly promising in studies of complex solar phenomena. As an example, we show a preliminary analysis of the 16 March 2016 C2.2 eruptive flare. Before this event, maintenance of SRH systems was carried out. Antennas were pointed at the Sun at about 06:36 UT. SRH observed this event at $6 \mathrm{GHz}$ with an interval of about $1 \mathrm{~s}$ between the images.

Figure 10 presents a prominence eruption near the west limb, which was observed in the $304 \AA$ channel of the Atmospheric Imaging Assembly (AIA) [Lemen et al., 2012] of the SDO. Arcs of different styles in Figure 10, $a-d$ outline the upper edge of the ascending prominence, and the dashed curve in Figure 10, $e$ represents the fit of its acceleration, which is up to $1.8 \mathrm{~km} / \mathrm{s}^{2}$.

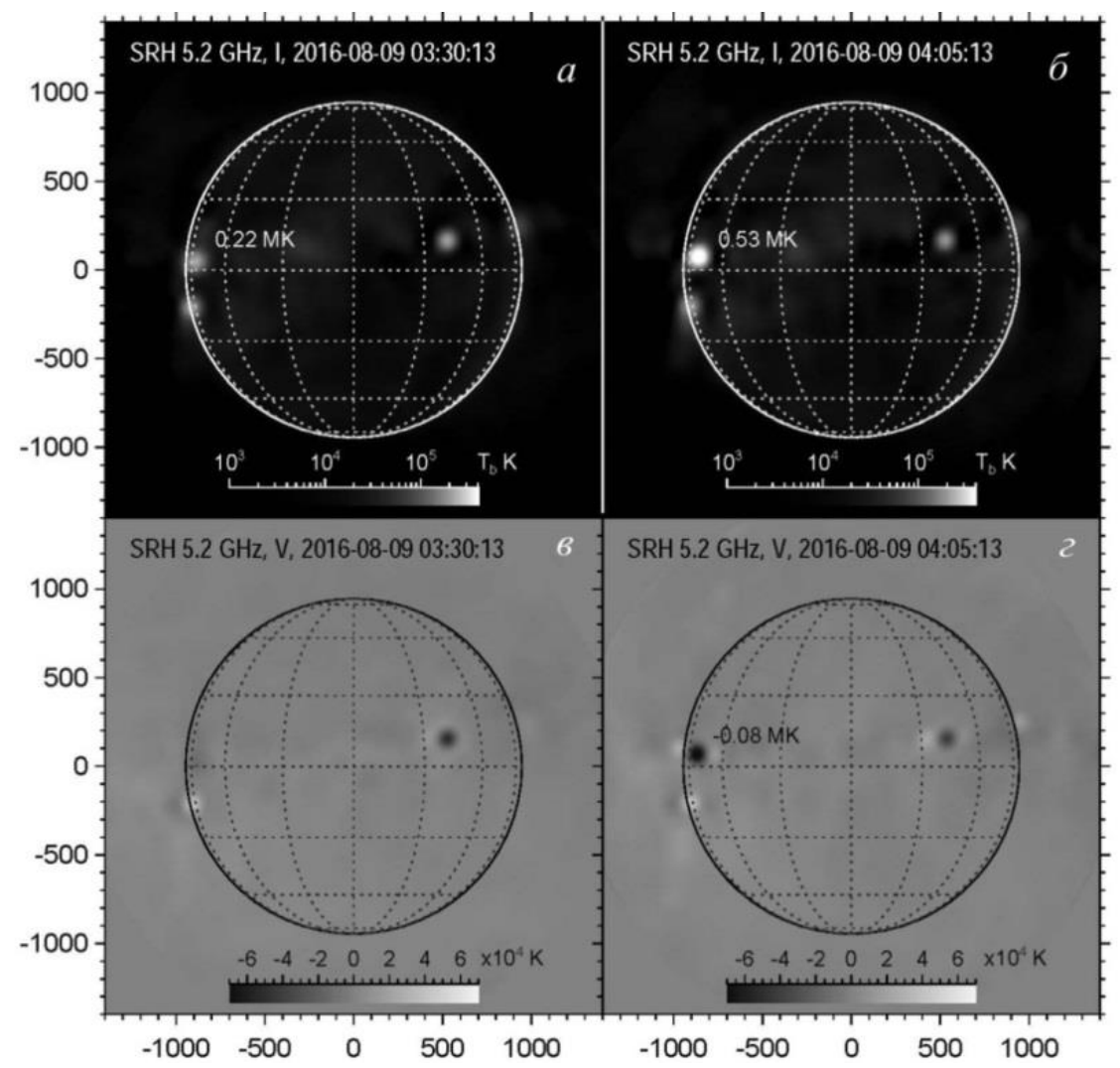

Figure 4. Solar radio images obtained by SRH at $5.2 \mathrm{GHz}$ in total intensity and polarization after cleaning procedure during the first negative burst (left) and after it (right). The negative burst is related to the screening of the northern source on the east limb. The axes show arc seconds from the solar disk center 


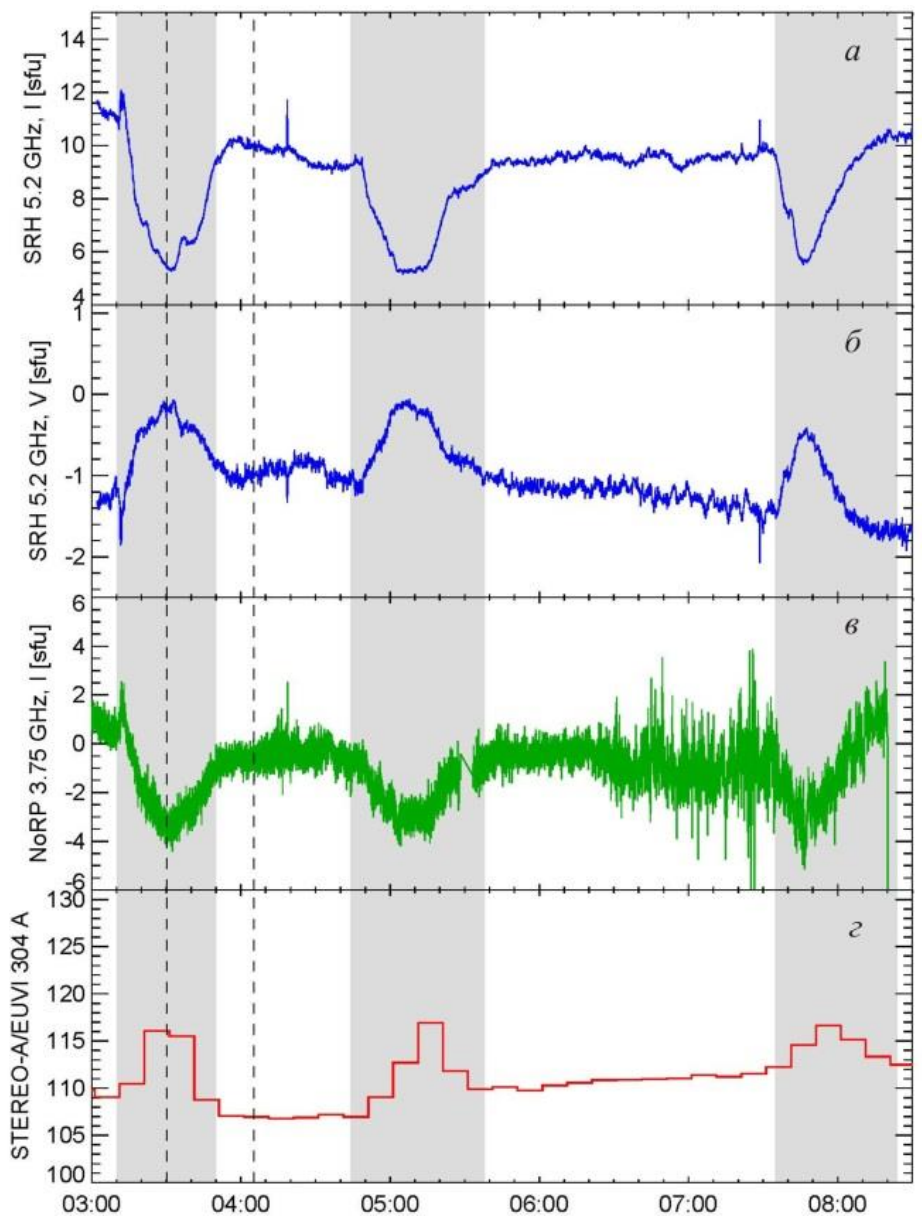

Figure 5. Observations on 9 August 2016. Time profiles in total intensity (a) and polarization (b) of the screened source computed from SRH images at $5.2 \mathrm{GHz}$ along with a total flux recorded by NoRP at $3.75 \mathrm{GHz}(c)$ and the ultraviolet flux in $304 \AA$ for the framed region in Figure $6(d)$. Vertical dashed lines indicate the observation times in Figure 4. Gray vertical shadings denote the intervals to which images in Figure 6 correspond

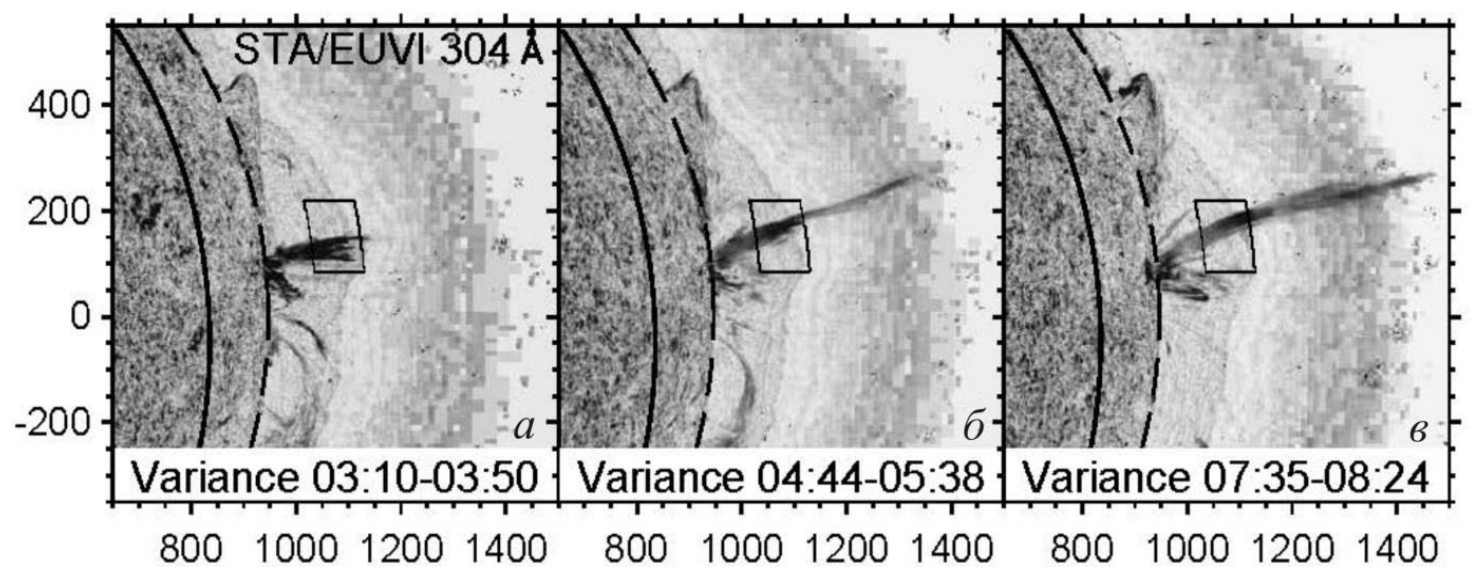

Figure 6. Observations on 9 August 2016. Images of three surges revealed by the variance analysis of STEREO-A/EUVI $304 \AA$ images in specified intervals. The frame at the center of the image indicates the region used to construct the time profiles in Figure 5, $d$. The dashed arc denotes the solar limb visible from STEREO-A. The thick solid arc corresponds to the east limb visible from Earth. The axes show arc seconds from the center of the solar disk as seen from STEREO-A 


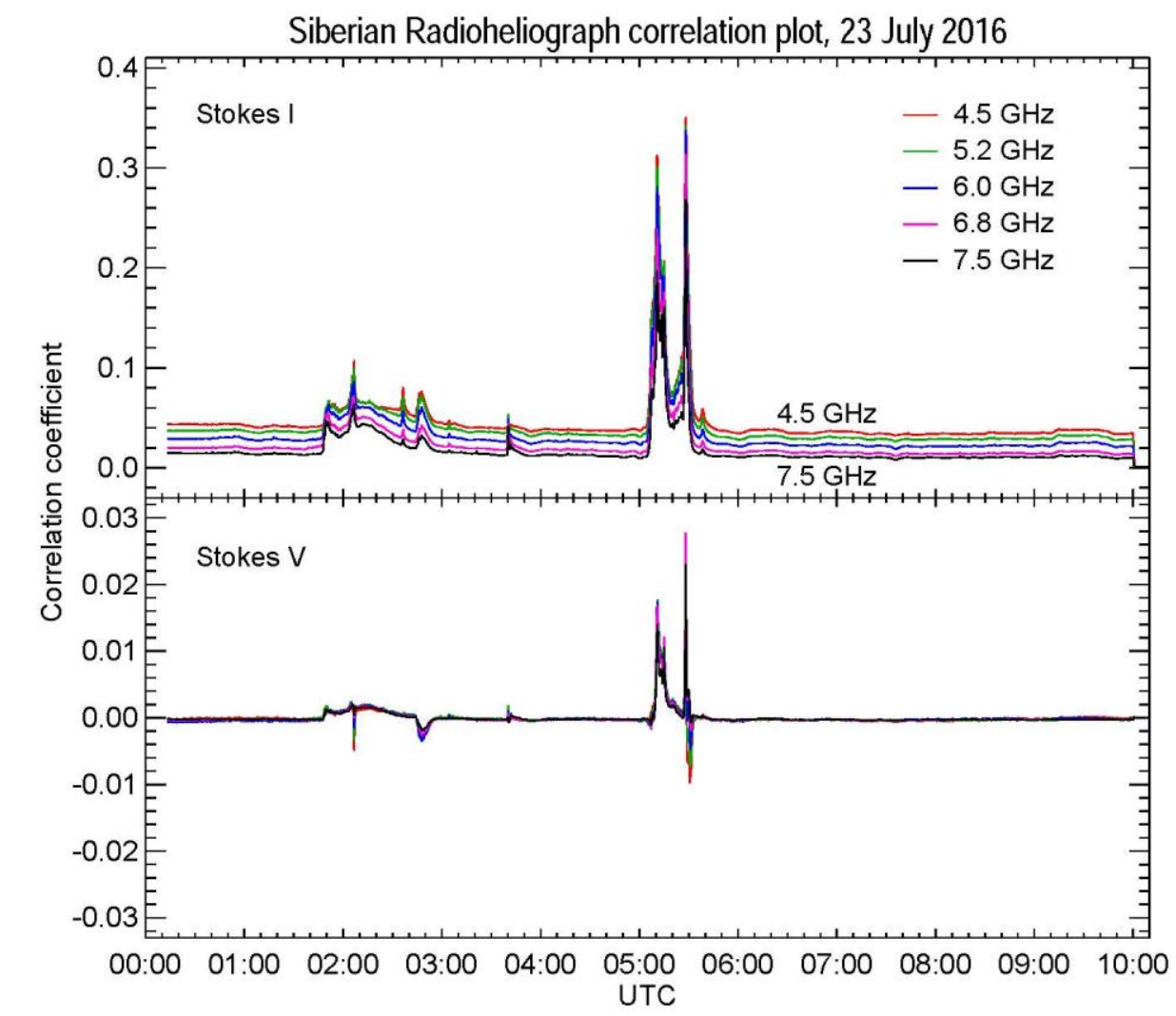

Figure 7. Correlation plots with powerful flares recorded on 23 July 2016

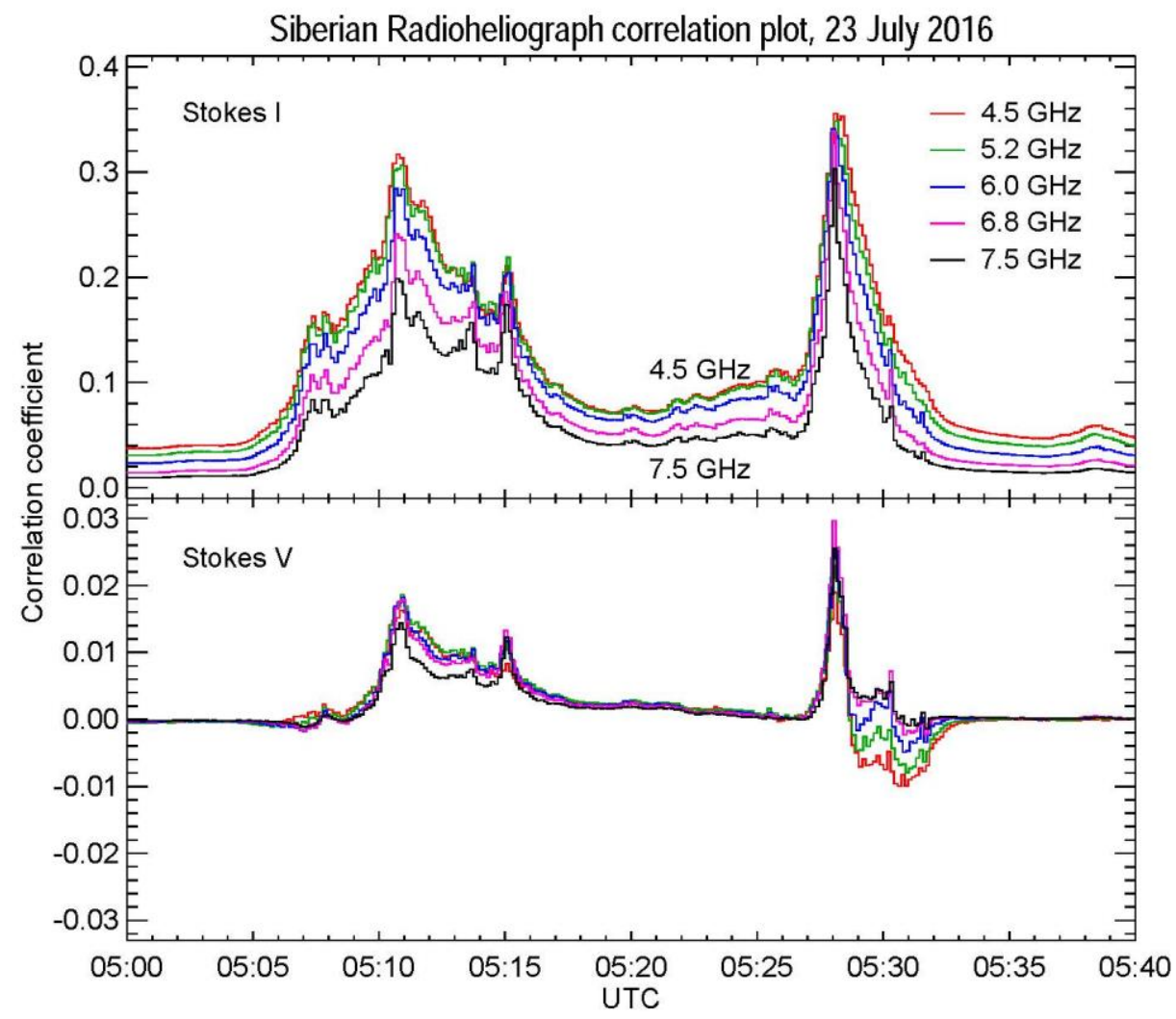

Figure 8. Correlation plots of two flares on 23 July 2016 with polarization reversal within the SRH frequency band (05:28:30-05:30:30 UT) 


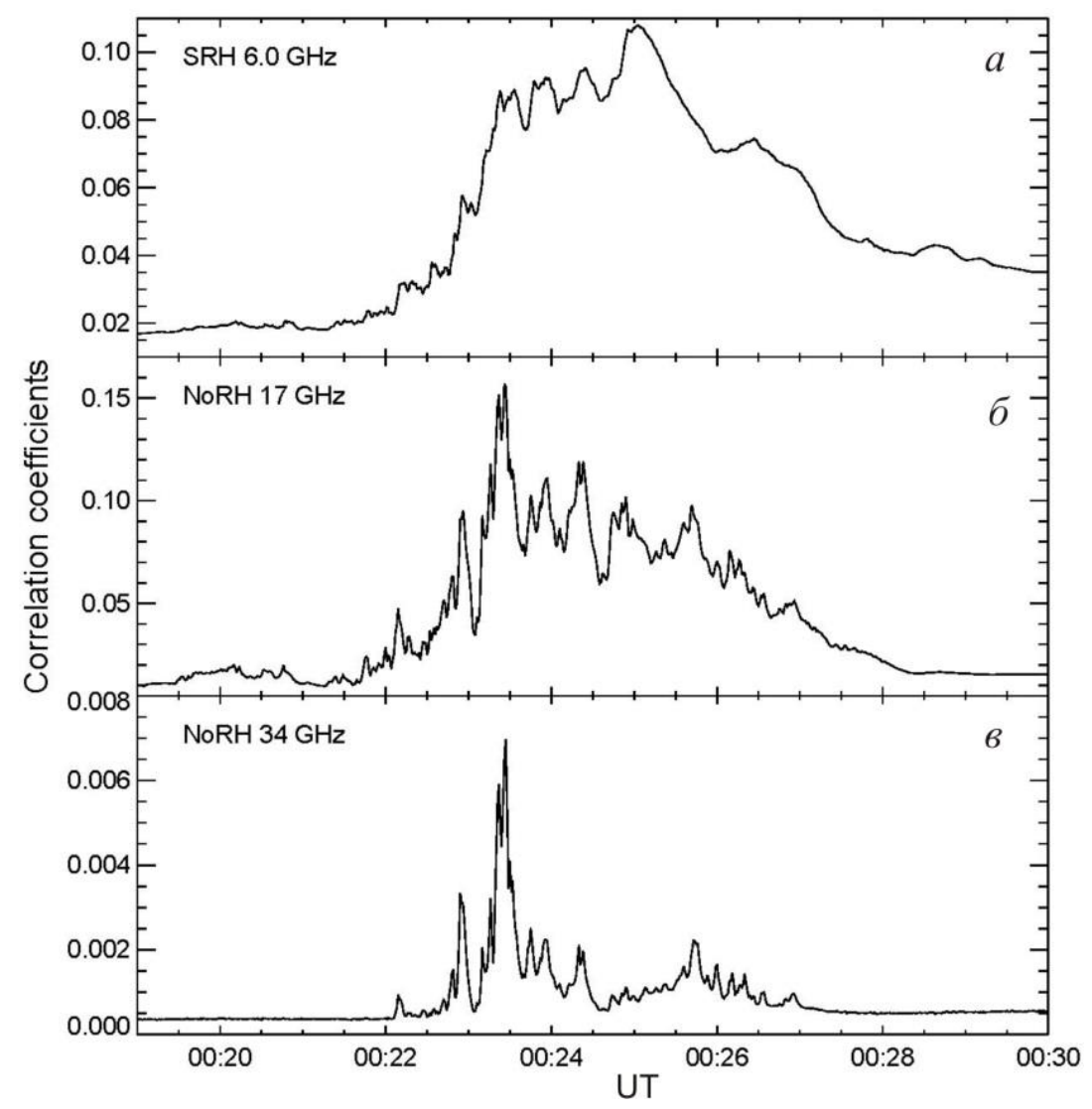

Figure 9. Correlation plots of the 18 April 2016 M7.6 flare recorded by SRH at $6 \mathrm{GHz}$ (a) and NoRH at 17 $\mathrm{GHz}(\mathrm{b})$ and $34 \mathrm{GHz}(\mathrm{c})$

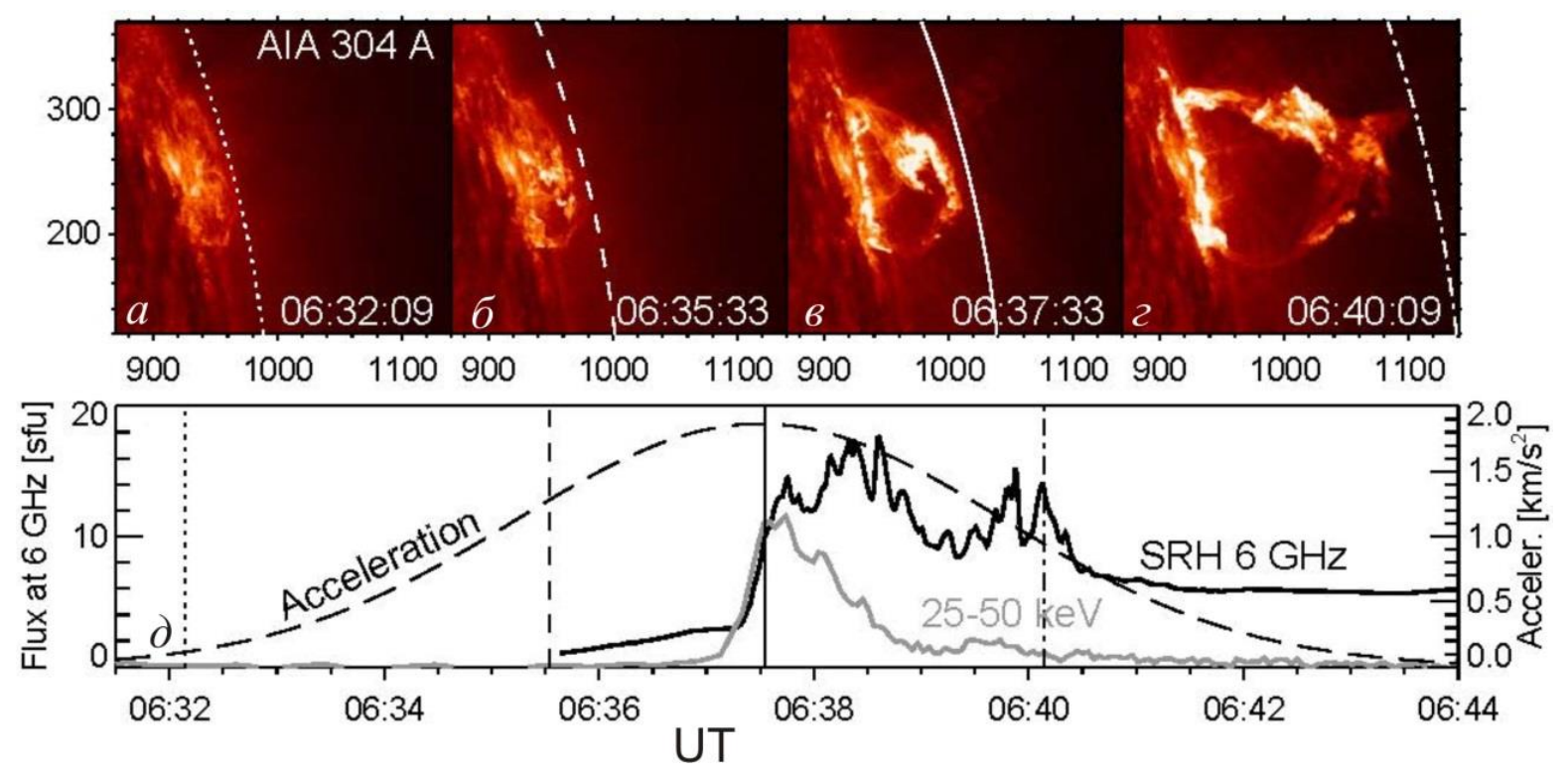

Figure 10. Eruptive flare of 16 March 2016 Top: prominence eruption in SDO/AIA $304 \AA$ images. The axes show arcseconds from the solar disk center. Arcs outline the upper edge of the rising prominence. Bottom: time profiles of the microwave (black, SRH $6 \mathrm{GHz}$ ) and hard X-ray (gray, Fermi 25-50 keV) emissions along with the measured acceleration of the prominence (dashed curve) corresponding to the arcs on panels $a-d$. Times of these images are marked on the bottom panel by vertical lines of the same styles as the arcs 
For comparison, Figure 10, $e$ also presents time profiles of hard X-rays recorded by the Fermi Gamma-Ray Burst Monitor [Meegan et al., 2009] and of the microwave burst computed from SRH images at 6.0 GHz. The prominence started to ascend a few minutes before the sharp onset of the burst in hard X-rays and microwaves. Acceleration of most electrons up to high energies in the flare, which are responsible for the hard X-ray and microwave burst, was clearly caused by the prominence eruption. This sequence of events is typical of eruptive flares [Grechnev et al., $2015,2016]$.

Figure 11 shows time profiles of the flare in different spectral ranges: in two hard X-ray channels (Fermi/GBM, $a$ ), in microwaves at $6 \mathrm{GHz}(\mathrm{SRH}, b)$, and in extreme ultraviolet (SDO/AIA $193 \AA, c)$. The last time profile is calculated from running difference images (the image observed $48 \mathrm{~s}$ before each current image is subtracted from it) within bright regions above flare ribbons $\leq 4$ " wide in the plane of the sky, i.e. at a height up to $3000 \mathrm{~km}$ (these regions are also seen in $304 \AA$ images in Figure 10,a,d). The temperature sensitivity region of the $193 \AA$ channel starts from $0.2 \mathrm{MK}\left(>2 \cdot 10^{5} \mathrm{~K}\right)$. Consequently, the time profile in Figure 11, $c$ represents hot dense regions above the flare ribbons whose prolonged emission is suppressed in running differences. The similarity between the time profiles at $6 \mathrm{GHz}$ and in the $193 \AA$ channel leaves no doubt that they have common sources. There is no such similarity with the time profiles in the $193 \AA$ channel for each of the separate regions above the flare ribbons. Hence, the sources of microwave emission were distributed throughout the whole lengths of the ribbons, being most likely located in the lower parts of the flare arcade.

The similarity of the microwave burst with hard X-rays in Figure 11, $a$ is less pronounced than with the time profile in the $193 \AA$ channel. The rapid emission increase at $6 \mathrm{GHz}$ occurred almost simultaneously with the hard Xray increase, but a slow rise in microwave emission had started earlier. The initial part of the observations at $6 \mathrm{GHz}$ is less reliable because of inaccurate SRH antenna pointing at the onset of the event; yet a similar emission increase in the $193 \AA$ channel confirms correctness of the SRH data. The second peak after 06:39 is also identified in an emission band $25-50 \mathrm{keV}$, though being weaker than in microwaves, but it is not seen in harder X-rays 50-98 keV. This suggests that electrons in the second peak have a softer spectrum. The long-term background of the microwave burst is most likely the sum of thermal bremsstrahlung from plasma in the flare arcade and emission of accelerated electrons trapped in its loops. Despite the differences in microwave and hard X-ray emissions, these bursts and their individual structural details bear general similarity. The sources of hard X-rays were most probably distributed over the length of the flare ribbons too. This agrees with widely accepted model predictions.

A combined microwave spectrum of this event in Figure 12 inferred from data acquired by total-flux spectropolaimeters [Zhdanov, Zandanov, 2015] and by NoRP shows a series of 5-10 s pulses, whose spectral maximum frequencies were $<10 \mathrm{GHz}$. The spectrum widths of these impulsive bursts during the second peak (06:39:0006:40:30 UT) are within 2-3 GHz, being atypically narrow for gyrosynchrotron emission even for the soft spectrum of radiating electrons. The differences between the spectra of microwave pulses suggest the difference between their sources, thus confirming the conclusion about their location in different places above the flare ribbons. 


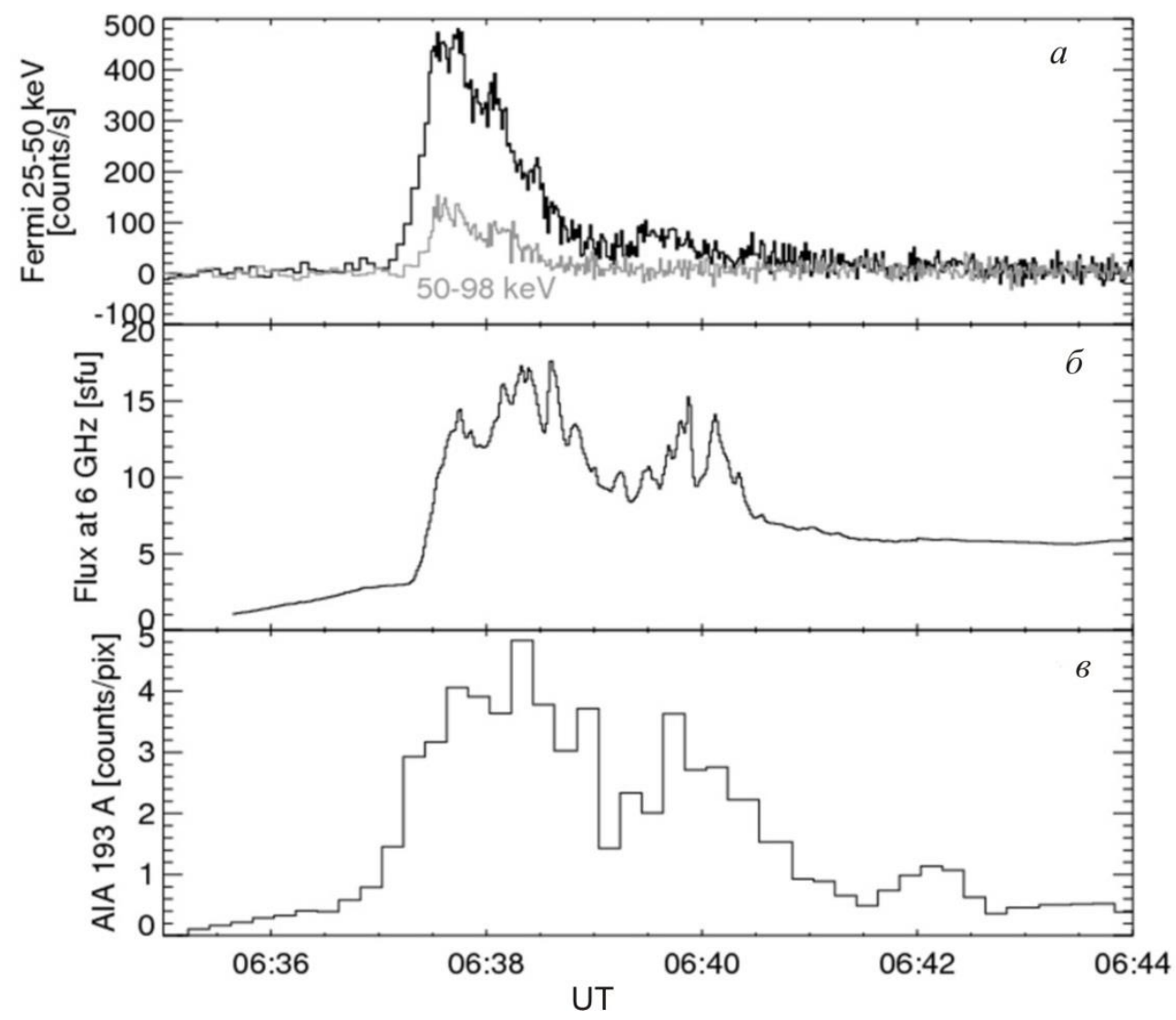

Figure 11. Time profiles of hard X-ray (Fermi/GBM, a), microwave (SRH, b), and extreme-ultraviolet (SDO/AIA $193 \AA, c$ ) emissions for the 16 March 2016 flare. Each point of the time profile (c) was calculated from the difference between the current SDO/AIA $193 \AA$ image and the image observed $48 \mathrm{~s}$ earlier within bright regions above the flare ribbons

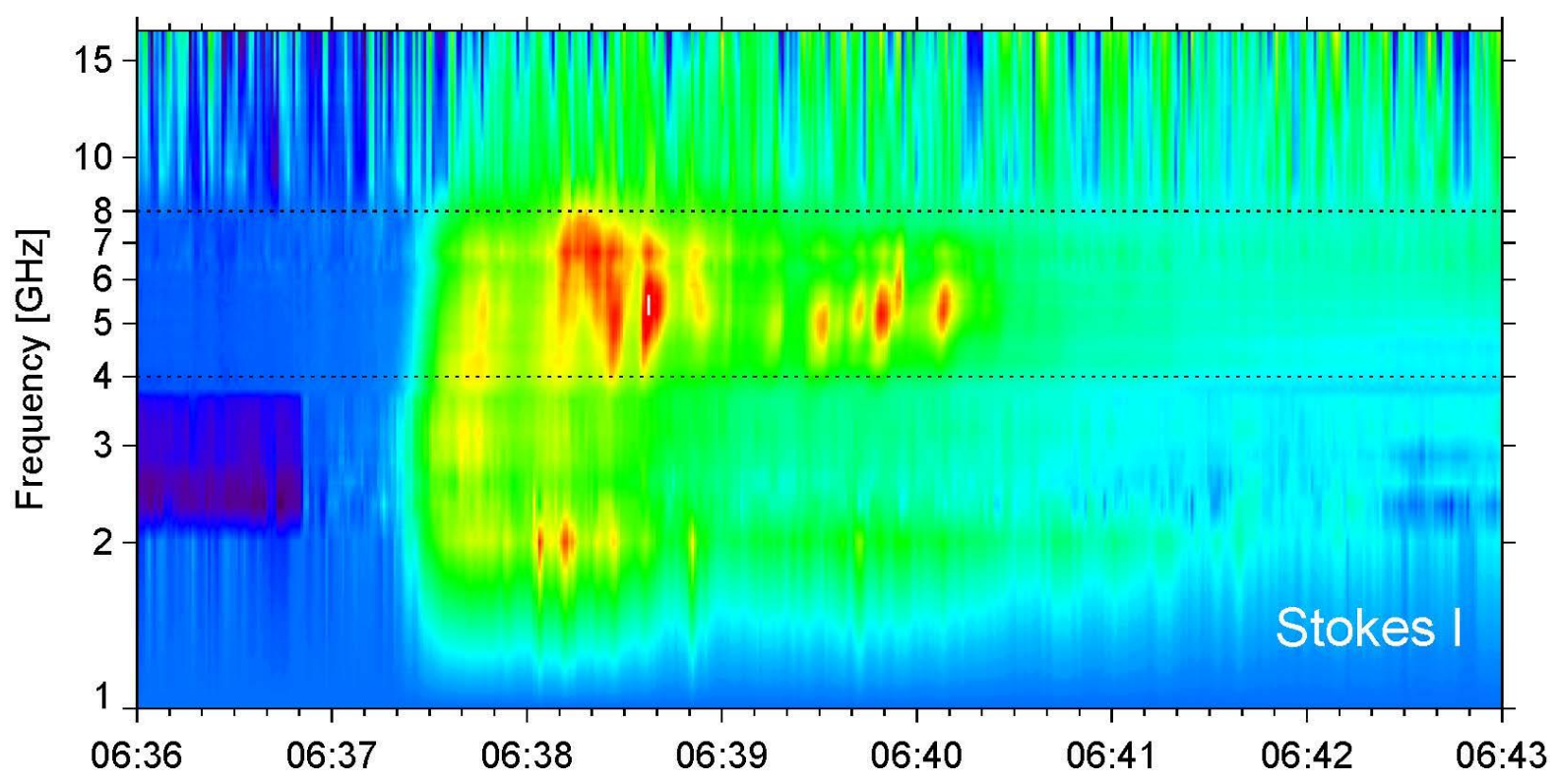

Figure 12. Dynamic spectrum of the 16 March 2016 flare. Horizontal dotted lines indicate the SRH frequency band 
Another feature of this flare is a modest microwave flux ( $\leq 18 \mathrm{sfu})$ with a rather y intense burst in hard X-rays. One of the causes of the low microwave emission could be compactness of its sources.

The preliminary analysis of the 16 March 2016 eruptive flare observed by SRH-48 shows its capabilities even with incomplete antenna array reducing its current spatial resolution. The joint analysis of the SRH and spectropolarimeter data with observations in different spectral ranges provides insight into the relationship between eruptions and flares and into properties of flare configurations even though their being unresolved. Observations made using instruments with limited spatial resolution and dynamic range suggest that non-thermal processes predominate in simple one- or two-loop flare configurations. This is difficult to reconcile with observations of emission of other types (e.g., in extreme ultraviolet) and with well-known models. The correspondence between various flare manifestations observed in different ranges in the event overviewed is in agreement with models and conclusions of recent studies [e.g., Grechnev et al., 2017].

\section{CONCLUSION}

Observations made using the first stage of the multiwave Siberian Radioheliograph demonstrate efficiency of design ideas and their accomplishment. The T-shaped antenna array with redundant baselines enables the implementation of fast and effective algorithms for solar imaging without the need of reference observations of other cosmic sources. The high sensitivity of the interferometer $\left(\approx 10^{-2} \mathrm{sfu}\right)$ combined with a wide dynamic range allows observing compact sources of powerful solar bursts without attenuators. Advantages of SRH are as follows: the temporal resolution high enough to study many processes (up to $0.56 \mathrm{~s}$ for both circularly-polarized components in the singlefrequency mode), multi-frequency observations with a tunable frequency set depending on observational program, image synthesis with optimization of required parameters (for example, spatial resolution or sensitivity), and the absence of geometric distortions from which SSRT images suffered.

SRH allows one to synthesize tens of thousands of solar images per day. Methods and software for synthesis and calibration of images and their subsequent analysis have been partially developed, in particular in our previous studies. They should be elaborated and adapted to address diverse observational and research issues.

Expansion of the antenna array up to 96 elements would be the next milestone in upgrading SRH. The spatial resolution of SRH-96 would be as high as 15". Additional antennas have already been installed, in particular in outer SSRT posts, which are located $330 \mathrm{~m}$ away from the center of the array. As SSRT observations showed, such resolution would be sufficient to study processes of initiation of coronal mass ejections and their propagation up to heights of one to two solar radii, thus filling the gap between observations in ultraviolet and optical ranges [Uralov et al., 2002, Alissandrakis et al., 2013]. Multi-frequency observations of active regions would provide important verification of coronal magnetography methods [Nita et al., 2011].

The first results presented here show a high potential of the new instrument, being related to only a few observational issues. The preliminary analysis of the events discussed needs to be completed. Some mentioned observational results require understanding. At the same time, ongoing SRH observations provide new information. Any interest in the development of the SRH software and data analysis is welcome. 
We are grateful to P.M. Svidsky for useful discussions. This work was supported by Fundamental Research Program 7 of the RAS Presidium "Experimental and theoretical studies of objects in the solar system and planetary systems of stars. Transitional and explosive processes in astrophysics", by RFBR under grants 15-02-01089 A and 15-02-03717 A, and by FASO under the project "Investigation into extremely weak solar activity in the microwave range" performed at SSRT. Experimental data were acquired using SSRT.

\section{REFERENCES}

Alissandrakis C.E., Kochanov A.A., Patsourakos S., Altyntsev A.T., Lesovoi S.V., Lesovoya N.N. Microwave and EUV Observations of an Erupting Filament and Associated Flare and Coronal Mass Ejections. Publications of the Astronomical Society of Japan. 2013, vol. 65, no. SP1, article id. S8, 10 p.

Altyntsev A.T., Kashapova L.K. Vvedenie v radioastronomiyu Solntsa [Introduction to Solar Radio Astronomy]. Irkutsk, IGU Publ., 2014. 203 p. (In Russian).

Altyntsev A., Meshalkina N., Meszarosova, H., Karlicky M., Palshin V., Lesovoi S. Sources of quasi-periodic pulses in the flare of 18 August 2012. Solar Phys. 2016, vol. 291, iss. 2, pp. 445-463.

Bastian T.S., Gary D.E., White S.M., Hurford G.J. Toward a frequency-agile solar radiotelescope. 18 NSO/Sacramento Peak Summer Workshop “Synoptic Solar Physics”. Sunspot, New Mexico 8-12 September 1997, p. 563 (ASP Conference Series. 1998, vol. 140).

Bogod V.M., 2011, RATAN-600 radio telescope in the 24th solar-activity cycle. I. New opportunities and tasks. Astrophys. Bull. 2011, vol. 66, iss. 2, pp. 190-204.

Borovik V.N. Quiet Sun from multifrequency radio observations on RATAN-600. Lecture Notes in Phys. 1994, vol. 432, pp. 185-190.

Fleishman G.D., Pal'shin V.D., Meshalkina N.S., Lysenko L., Kashapova L.K., Altyntsev A.T. A cold flare with delayed heating. Astrophys. J. 2016, vol. 822, iss. 2, article id. 71, 20 p.

Gary D.E., Nita G.M., Sane N. Expanded Owens Valley Solar Array (EOVSA) testbed and prototype. American Astronomical Society, AAS Meeting 220, id. 204.302012.

Grechnev V.V. A method to analyze imaging radio data on solar flares. Solar Phys. 2003, vol. 213, iss. 1, pp. 103-110.

Grechnev V.V., Lesovoi S.V., Smolkov G.Ya., Krissinel B.B., Zandanov V.G., Altyntsev A.T., Kardapolova N.N., Sergeev R.Y., Uralov A.M., Maksimov V.P., Lubyshev B.I. The Siberian Solar Radio Telescope: The current state of the instrument, observations, and data. Solar Phys. 2003, vol. 216, iss. 1, pp. 239-272.

Grechnev V.V., Kuz'menko I.V., Uralov A.M., Chertok M., Kochanov A.A. Microwave negative bursts as indications of reconnection between eruptive filaments and a large-scale coronal magnetic environment. Publications of the Astronomical Society of Japan. 2013, vol. 65, no. SP1, article id. S10, 9 p.

Grechnev V.V., Uralov A.M., Kuzmenko I.V., Kochanov A.A., Chertok I.M., Kalashnikov S.S. Responsibility of a filament eruption for the initiation of a flare, CME, and blast wave, and its possible transformation into a bow shock. Solar Phys. 2015, vol. 290, iss. 1, pp. 129-158.

Grechnev V.V., Uralov A.M., Kochanov A.A., Kuzmenko I.V., Prosovetsky D.V., Egorov Y.I., Fainshtein V.G., Kashapova L.K. A tiny eruptive filament as a flux-rope progenitor and driver of a large-scale CME and wave. Solar Phys. 2016, vol. 291, iss. 4, pp. 1173-1208.

Grechnev V.V., Uralov A.M., Kiselev V.I., Kochanov A.A. The 26 December 2001 solar eruptive event responsible for GLE63. II. Multi-loop structure of microwave sources in a major long-duration flare. Solar Phys. 2017, vol. 292, iss. 1, article id. 3, 27 p.

Kaltman T.I., Kochanov A.A., Myshyakov I.I., Maksimov V.P., Prosovetsky D.V., Tokhchukova S.K. Observations and modeling of the spatial distribution and microwave radiation spectrum of the active region NOAA 11734. Geomagnetism and Aeronomy. 2015, vol. 55, iss. 8, pp. 1124-1130. 
Kochanov A.A., Anfinogentov S.A., Prosovetsky D.V., Rudenko G.V., Grechnev V.V. Imaging of the solar atmosphere by the Siberian Solar Radio Telescope at $5.7 \mathrm{GHz}$ with an enhanced dynamic range. Publications of the Astronomical Society of Japan. 2013, vol. 65, no. SP1, article id. S19, 12, p.

Lang K.R., Willson R.F., Kile J.N., Lemen J., Strong K.T., Bogod V.M., Gelfreikh G.B., Ryabov B.I., Hafizov S.R., Abramov V.E., Tsvetkov S.V. Magnetospheres of solar active regions inferred from spectral-polarization observations with high spatial resolution. Astrophys. J. 1993, vol. 419, pp. 398-417.

Lemen J.R., Title A.M., Akin D.J., et al. The Atmospheric Imaging Assembly (AIA) on the Solar Dynamics Observatory (SDO). Solar Phys. 2012, vol. 275, iss. 1, pp. 17-40.

Lesovoi S.V., Altyntsev A.T., Ivanov E.F., Gubin A.V. A 96-antenna radioheliograph. Res. Astron. Astrophys. 2014, vol. 14 , no. 7, pp. 864-868.

Lesovoi S.V., Kobets V.S. Correlation curves of the Siberian Radioheliograph. Solnechno-zemnaya fizika [Solar-Terrestrial Phys.]. 2017, vol. 3, no. 1, pp. 17-21. (In Russian).

Meegan C., Lichti G., Bhat P.N., Bissaldi E., Briggs M.S., Connaughton V., Diehl R., Fishman G., Greiner J., Hoover A.S., van der Horst A.J., von Kienlin A., Kippen R.M., Kouveliotou C., McBreen S., Paciesas W.S., Preece R., Steinle H., Wallace M.S., Wilson R.B., Wilson-Hodgeet C. The Fermi Gamma-ray Burst Monitor. Astrophys. J. 2009, vol. 702, no. 1, pp. $791-804$.

Nakajima H., Nishio M., Enome S., Shibasaki K., Takano T., Hanaoka Y., Torii C., Sekiguchi H., Bushimata T., Kawashima S., Shinohara N., Irimajiri Y., Koshiishi H., Kosugi T., Shiomi Y., Sawa M., Kai K. New Nobeyama Radio Heliograph. J. Astrophys. Astron. Suppl. 1995, vol. 16, pp. 437-442.

Nita G.M., Fleishman G.D., Jing, Ju, Lesovoi S.V., Bogod V.M., Yasnov L.V., Wang H., Gary D.E. Three-dimensional structure of microwave sources from solar rotation stereoscopy versus magnetic extrapolations. Astrophys. J. 2011, vol. 737, iss. 2, article id. $82,12 \mathrm{p}$.

Nita G.M., Gary D.E., Lee J. Statistical study of two years of solar flare radio spectra obtained with the Owens Valley Solar Array. Astrophys. J. 2004, vol. 605, iss. 1, pp. 528-545.

Smolkov G.Ya., Pistolkors A.A., Treskov T.A., Krissinel B.B., Putilov V.A. The Siberian Solar Radio-Telescope — Parameters and principle of operation, objectives and results of first observations of spatio-temporal properties of development of active regions and flares. Astrophys. Space Sci. 1986, vol. 119, no. 1, pp. 1-4.

Uralov A.M., Lesovoi S.V., Zandanov V.G., Grechnev V.V. Dual-filament initiation of a coronal mass ejection: Observations and model. Solar Phys. 2002. vol. 208, iss. 1, pp. 69-90.

Wang Z., Gary D.E., Fleishman G.D., White S.M. Coronal magnetography of a simulated solar active region from microwave imaging spectropolarimetry. Astrophys. J. 2015, vol. 805, iss. 2, article id. 93, 13 p.

Yan Y., Zhang J., Wang W., Liu F., Chen Z., Ji G. The Chinese Spectral Radioheliograph - CSRH. Earth, Moon, and Planets. 2009, vol. 104, iss. 1-4, pp. 97-100.

Zhdanov D.A., Zandanov V.G. Observations of microwave fine structures by the Badary broadband microwave spectropolarimeter and the Siberian Solar Radio Telescope. Solar Phys. 2015, vol. 290, pp. 287-294.

Zirin H., Baumert B.M., Hurford G.J. The microwave brightness temperature spectrum of the quiet Sun. Astrophys. J. Part 1 (ISSN 0004-637X). 1991, vol. 370, pp. 779-783.

http://badary.iszf. irk.ru (accessed December 10, 2016)

ftp://badary.iszf.irk.ru/data/srh48 (accessed December 10, 2016).

$\mathrm{ftp} / / /$ badary.iszf.irk.ru (accessed December 10, 2016).

http://badary.iszf.irk.ru/srhCorrPlot.php (accessed December 10, 2016). 